Journal of Mathematics and Informatics

Vol. 18, 2020, 33-44

ISSN: 2349-0632 (P), 2349-0640 (online)

Published 20 January 2020

www.researchmathsci.org

DOI: http://dx.doi.org/10.22457/jmi.v18a3146

Journal of

Mathematics and

Informatics

\title{
Computational Fluid Dynamics of Manhole Overflow Due to Storm-water Inflow
}

\author{
Sifa Yohana Baseka ${ }^{1}$ and Verdiana Grace Masanja ${ }^{2}$ \\ ${ }^{1}$ School of Computational and Communication Science and Engineering \\ Nelson Mandela African Institute of Science and Technology P.O.Box 447 \\ Arusha, Tanzania. \\ Email: basekas@nm-aist.ac.tz; lumbelumbelumbe@gmail.com \\ ${ }^{2}$ School of Computational and Communication Science and Engineering \\ Nelson Mandela African Institute of Science and Technology P.O. Box 447 \\ Arusha, Tanzania. Email: verdiana.masanja@nm-aist.ac.tz \\ ${ }^{1}$ Corresponding author.
}

Received 10 November 2019; accepted 30 November 2019

\begin{abstract}
In this study, the storm water overflow on manholes is numerically solved. To produce a real representation of storm overflow, the Navier-Stokes equations were used. Turbulence was modelled using the standard k-epsilon turbulence model together with the volume of fluid method for phase surface tracking. The open-source computational fluid dynamics (CFD) tool OpenFOAM 5.0 was used for solving the model while the visualization tool, paraview 5.4.0, was used for processing the solution data. The convergence test was performed at three different mesh sizes. The numerical solution established mesh independence by producing similar results at varied meshes sizes. The risen storm-water column inside the manhole exerts a non-uniform pressure on the manhole cover. The non-uniform pressure distribution leads to different uplifting forces at manhole cover areas. However, the global uplifting force remains constant as long as the storm water overflow is occurring on the manhole. The overflow intensity on the manhole is not the same, areas opposite to the outlet pipe provide the highest intensity of the leaking storm-water.
\end{abstract}

Keywords: manhole overflow, storm-water inflow, global force, storm-water column

\section{AMS Mathematics Subject Classification (2010): 37N10}

\section{Introduction}

Management of urban wastewater is crucial to the protection of people's health and environmental sanitation. It is related to various challenges such as sewer overflow which contributes much to the spilling of wastewater to the environment, highly noted during rainfall. People's residences, commercial areas like markets, institutional areas and industrial areas are the main producers of urban wastewater [1]. The wastes are collected and transported through existing urban sewer networks which are composed of different 


\section{Sifa Yohana Baseka and Verdiana Grace Masanja}

elements such as sewer pipes, septic tanks, force mains, treatment plants, manholes and waste disposal areas. Each component of the sewer network is crucial for instance the changing of the chemical behavior of wastewater which occurs in treatment plants to reduce or eliminate health and environment problems upon the wastewater discharge [2]. Various studies have been undertaken in the field of hydraulic features of wastewater and storm-water flow within manholes. [3], investigated the physics behind vibration of the manhole cover. The author considered this to be a nonlinear spring-mass system, whose model gave a lot of information on the mechanism of manhole cover vibrations. [4] considered the air pressurization from air pockets in the water column inside the manhole and the rising water level inside the manhole to have the ability to create the displacement of the manhole cover. Air pressurization and the rising water level are the two flow conditions that are associated with displacement of the manhole cover. [5] and [6] developed a physical model for investigating the head loss coefficient in a four-way manhole and a gully-manhole. The calculated coefficient was used to propose a head loss coefficient equation that can be used in the simulation of flow features for pipes and surface flow [7], proposed a way of reducing the head loss coefficient in manhole as it is related to passage complications. The author recommended that half rectangular benching designs in circular manholes, and full rectangular benching designs in square manholes, should be used for the best reduction of the head loss coefficient. In working with manhole uplift due to soil liquefaction, [8] suggested the inclusion of interface friction forces regardless of their small magnitude.

Despite efforts done in solving problems related to sewer systems, still, some challenges continue to exist such as leakage of wastewater from the system. Little attention has been paid to the understanding of overflow dynamics through manholes under the contribution of water during rainfall. It was the aim of this study to use CFD to model the wastewater overflow on manholes due to storm-water inflow into sewer systems during heavy rainfall and investigate the pressure head uplifting forces to the manhole cover.

\section{Model formulation}

The model formulation was associated with sewage overflow phenomenon on the manhole, due to storm-water inflow into sewer systems lines during heavy rainfall. The governing equations providing the basis for modelling of an incompressible, Newtonian and isothermal fluid flow was the Reynolds Averaged Navier-Stokes (RANS) equations, [9]. These equations express the conservation of mass and conservation of linear momentum on the turbulent flow that must be satisfied for each control volume of the computational domain. On vector notation, the Reynolds Averaged Navier-Stokes equations are given in Eqn. (1) and Eqn. (2),

$$
\begin{aligned}
& \nabla \cdot \vec{u}=0 \\
& \frac{\partial \vec{v}}{\partial t}+\vec{u} \cdot(\nabla \vec{u})=-\nabla \vec{p}+\nabla \cdot \tau+\vec{g}
\end{aligned}
$$

with the mean velocity vector $\vec{u}$, the gravitational vector $\vec{g}$ acting on the flow and mean kinematic pressure $p$, the ratio between mean pressure and density. The term containing gravitational acceleration represents the weight per unit volume as the body force acting 
Computational Fluid Dynamics of Manhole Overflow Due to Storm-water Inflow on the moving fluid. The components of mean shear stress tensor $\tau_{i j}$, for $i, j$ taking on values 1, 2, 3, are given by Eqn. (3) with $\mu$ being the dynamic viscosity. The second term of the shear stress tensor represents the stress due to turbulent motion, the Reynolds stress term. It contains unknowns, the fluctuating velocity components $u_{i}^{\prime}$ that are further modelled by using the Boussinesq hypothesis, where Reynolds stress is proportional to the mean velocity gradient, Eqn. (4).

$$
\begin{array}{r}
\tau=\tau_{i j}=\mu\left(\frac{\partial u_{i}}{\partial x_{j}}+\frac{\partial u_{j}}{\partial x_{i}}\right)-\rho \overline{u_{i}^{\prime} u_{j}^{\prime}} \\
-\rho \overline{u_{i}^{\prime} u_{j}^{\prime}}=\mu_{t}\left(\frac{\partial u_{i}}{\partial x_{j}}+\frac{\partial u_{j}}{\partial x_{i}}\right)-\rho \frac{2}{3} k \delta_{i j}
\end{array}
$$

with $\rho$ the fluid density, $\delta_{i j}=\left\{\begin{array}{l}0 \text { for } i \neq j \\ 1 \text { for } i=j\end{array}\right.$-is the Kronecker delta. The turbulent viscosity $\mu_{t}$ is given by Eqn. (5). It depends on the kinetic energy $k$ and its dissipation rate $\in . C_{\mu}$, is the turbulent model constant equal to 0.09 .

$$
\mu_{t}=C_{\mu} \rho \frac{k^{2}}{\epsilon}
$$

RANS equations contain more unknowns than the number of equations [10], due to the Reynolds stresses. The k-epsilon $(k-\epsilon)$ is a two-equation model making a closure to the RANS equations for modelling turbulence stresses. It contains two transport equations; one for turbulent kinetic energy $k$ and another for the rate of dissipation of turbulent kinetic energy $\in$. The $k-\in$ values are obtained by solving the two transport equations which are used to find values for the turbulent dynamic viscosity $\mu_{t}$ in Eqn. (5). Eqn. (6) and Eqn. (7) are used to estimate the initial values for the turbulence kinetic energy and its dissipation rate.

$$
\begin{aligned}
& k=\frac{3}{2}(u I)^{2} \\
& \in=C \mu^{\frac{3}{4}} \frac{k^{\frac{3}{2}}}{l}
\end{aligned}
$$

with $C_{\mu}$ the turbulent model constant, $k$ is the turbulent kinetic energy, $\in$ the dissipation rate and $l$ is the turbulent length scale. $u$, is the mean velocity, $I$ is the turbulent intensity which depends on the Reynolds number Re, Eqn. (8). The model for the stormwater flow in the manhole is comprised of a set of partial differential equations Eqn. (9) 
Sifa Yohana Baseka and Verdiana Grace Masanja

to Eqn. (12) consisting of the RANS equations, the transport equations for turbulent kinetic energy $k$, and the dissipation rate $\in$.

$$
\begin{gathered}
I=0.16(\mathrm{Re})^{-0.125} \\
\nabla \cdot u=0 \\
\frac{\partial V}{\partial t}+u \cdot(\nabla u)=-\nabla p+\nabla \cdot \tau+g \\
\frac{\partial k}{\partial t}+u(\nabla k)=P^{(k)}-\epsilon+\nabla \cdot\left(\frac{v_{t}}{\sigma_{k}} \nabla k\right)+v \nabla \cdot(\nabla k) \\
\frac{\partial \epsilon}{\partial t}+u(\nabla \in)=-C_{\in 1} \frac{\epsilon}{k} P^{(k)}-C_{\in 2} \frac{\epsilon^{2}}{k}+\nabla \cdot\left(\frac{v_{t}}{\sigma_{\epsilon}} \nabla \in\right)+v \nabla \cdot(\nabla \in)
\end{gathered}
$$

$P^{(k)}$, is the production rate of turbulent kinetic energy per unit mass and $C_{\in 1}, C_{\in 2}$ $\sigma_{k}, \sigma_{\epsilon}$ are model constants.

\section{Numerical simulation}

The geometry for the computational domain simulating storm-water overflow is the manhole illustrated in (Figure 1). The computational domain was designed using a free source 3D modelling software, FreeCad 0.18. The manhole (the large rectangular container) has two circular pipes joined to it, the inlet pipe and the outlet pipe. The boundaries extracted from the geometry are the inlet boundary, the outlet boundary, and the wall boundary. There are two outlet boundaries, the outlet boundary at the outlet pipe and outlet boundary at the top of the manhole. The inlet boundary is located at the patch of the inlet pipe whereas the outlet boundary is located at the patch of the outlet boundary. The wall boundary includes all stationary walls remaining from the geometry setup. The mesh for the geometry setup is illustrated in Figure 2. The whole of the computational domain consists of 243,000 cells.

The boundary condition at the inlet is fixed value boundary condition. It is a Dirichlet boundary condition in OpenFOAM that allows the assignment of model variables' initial values to the inlet patch [11]. Initial conditions for the turbulent kinetic energy $k$, dissipation rate $\epsilon$, velocity $v$ and phase value, $\alpha$, are assigned using this boundary condition. The "calculated" boundary condition is used for the turbulent viscosity. It assigns the field value derived from other field values. The initial pressure field within the computational domain is defined by the total pressure boundary condition, Eqn. (13), for incompressible fluid flow, where $p$ denotes static pressure, $p_{0}$ the total pressure and the last term is the dynamic pressure due to kinetic energy of the moving fluid [12],

$$
p=p_{0}-\frac{1}{2} \rho u^{2}
$$


Computational Fluid Dynamics of Manhole Overflow Due to Storm-water Inflow

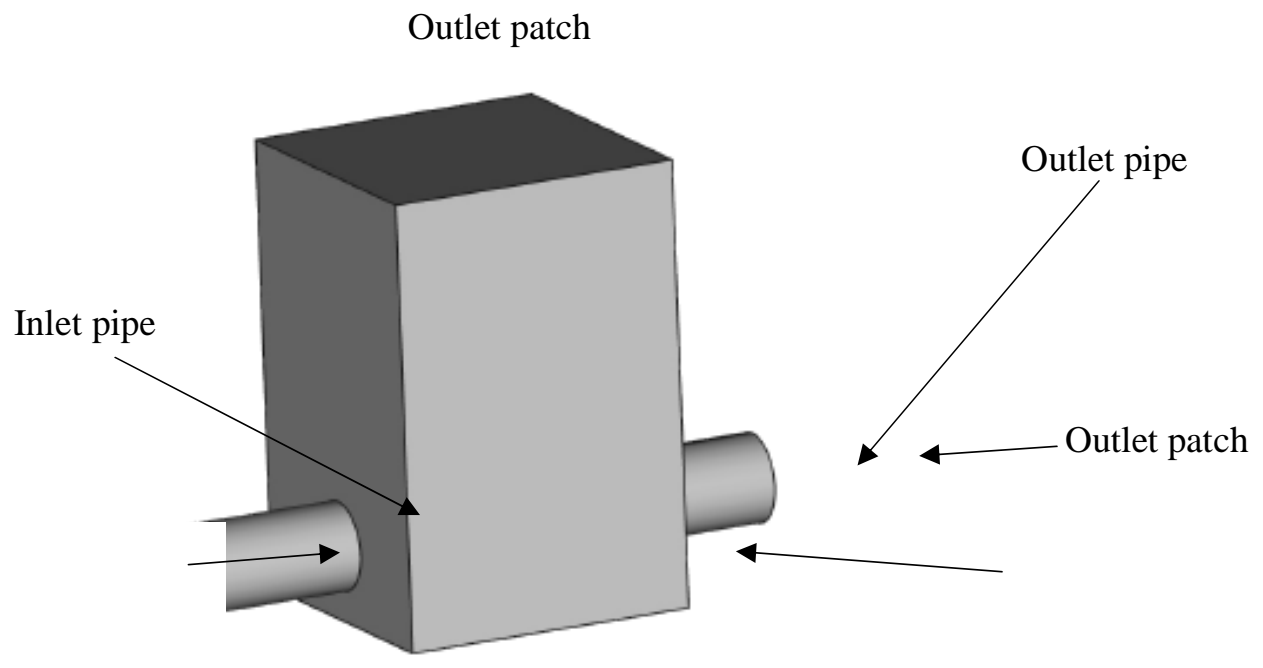

Figure 1: Manhole

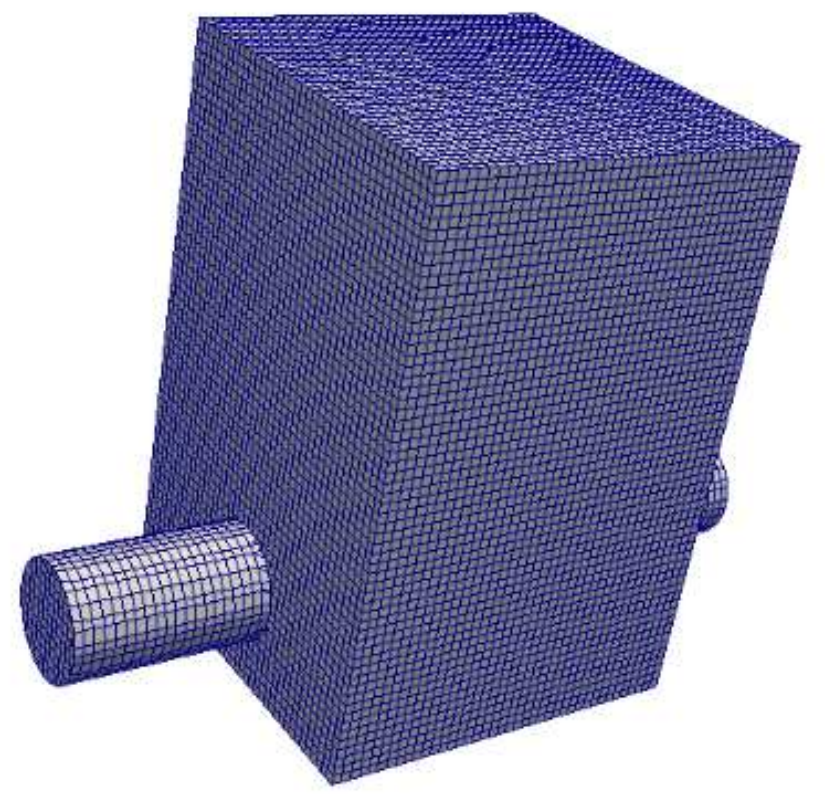

Figure 2: Mesh for the manhole

InletOutlet is a boundary condition in OpenFOAM used on the outlet patches for $k$ and $\in$. It is the zero gradient boundary condition that applies to the quantity field when the field is out of the domain. Also, it is a fixed value boundary condition as the field is flowing in the reverse direction. Since the quantity field at the outlet patch is unspecified, the zero gradient boundary condition sets the gradient of quantity field to zero, so that it 


\section{Sifa Yohana Baseka and Verdiana Grace Masanja}

can be extrapolated from the nearest cells. The InletOutlet boundary condition restricts the unwanted backflow of the quantity field through the value defined at the inlet value entry. For the velocity field at the outlet patch, pressure InletOutletVelocity boundary condition in OpenFOAMis used due to the initial internal pressure specification. The velocity field at a fluid-solid boundary is equal to the velocity field of the solid boundary[13]. The no Slip boundary condition in OpenFOAM was used to account for the zero velocity of the viscous fluid at the stationary walls in contact with the moving storm-water.

InterFoam, a multiphase solver in OpenFOAM 5.0 was used for simulation of the storm-water overflow of the manhole. The solver solves the continuity and Navier-Stokes equations for two incompressible, isothermal, immiscible, transient and turbulent fluids. The material properties such as the density, viscosity, and specific heat capacity are constant in the region filled by one of the two fluid phases except at the interphase [14]. To capture the interphase between the two phases, InterFoam employs the volume of fluid (VOF) method, Eqn. (14), a numerical technique for tracking and locating an interface between phases [15]. The model uses the volume fraction $\alpha$ to denote the individual phases. The liquid-gas interface arises within mesh cells where $\alpha$ take the value between 0 and1. The computational cell is completely filled with water at $\alpha=1$ and for $\alpha=0$ it is completely filled with air

$$
\frac{\partial \alpha}{\partial t}+\nabla \cdot(\alpha v)=0
$$

Incorporating the initial and boundary conditions, the model results for the manhole overflow is illustrated in (figure 3 ).

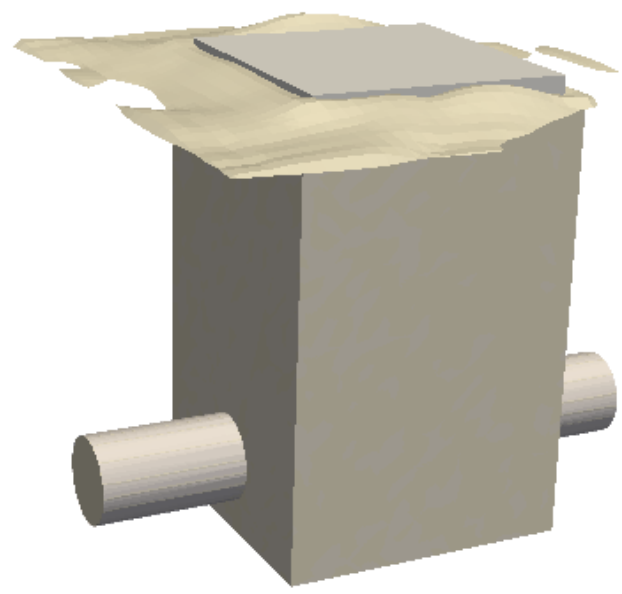

Figure 3: An overflowed manhole

\section{Results and discussion}

\subsection{Convergence test}

Convergence test was done at three different meshes to ensure the independence of the model results on the discretization. The first mesh contained 243,000cells, the second 
Computational Fluid Dynamics of Manhole Overflow Due to Storm-water Inflow

mesh 415,000 cells and the last mesh 600,000 cells. In order to converge, the model must give nearly the same results at different levels of mesh refinement. The pressure residual profile for the different meshes is shown in figure 4.

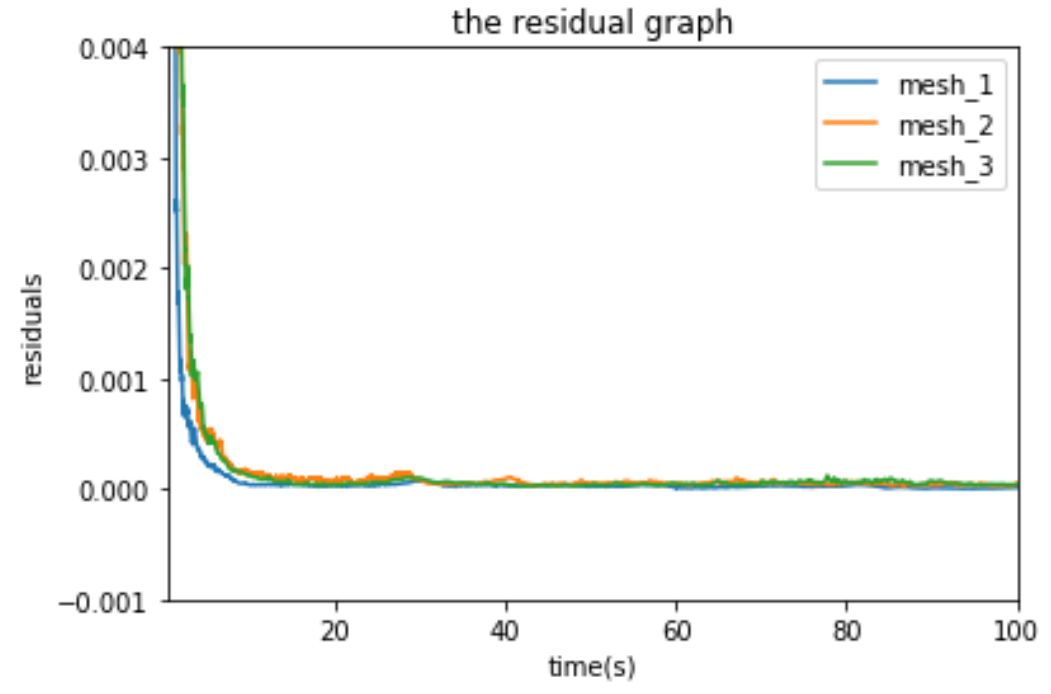

Figure 4: The pressure residuals for different meshes

From the graph, figure 4, it can be seen that as the residuals decrease with increasing time, curves of all the three meshes are converging to the same result until they attain the constant value. It can be observed that curves of the three different meshes are nearly coincident after attaining a steady solution, thus the difference between attained results by the three mesh sizes is negligible. Normally a finer mesh gives much better resolution on model results but it takes quite a lot of computational time. In order to reduce the computational time, the mesh of 243,000 cells was chosen to do the computations and simulations for the storm-water overflow in the manhole.

Numerical simulation for the sewer flow inside the manhole was compared with experimental results from two connected manholes setup at the University of Coimbra [16]. The setup consists of two connected manholes each with a diameter of $1 \mathrm{~m}$, and the inlet and outlet pipes each with $0.3 \mathrm{~m}$ diameter. The setup was used to measure pressure values at various points by using pressure sensors and measuring the height of the risen water column inside a manhole at different inflow rates of the water. After reaching a steady-state flow, the pressure values and water column height inside manhole were recorded. The numerical model results were compared to the experimental results at inflow rates of 451/s, 601/s, 901/s, 1001/s, and 1301/s. The curve output for the numerical (this study) and experimental ([16]) results are shown in figure 5. The red graph represents the experimental storm-water column values while the blue graph represents the numerical storm-water column values. It is observed in figure 5, that there is a good agreement between the experimental results and the numerical results of this study. 
Sifa Yohana Baseka and Verdiana Grace Masanja

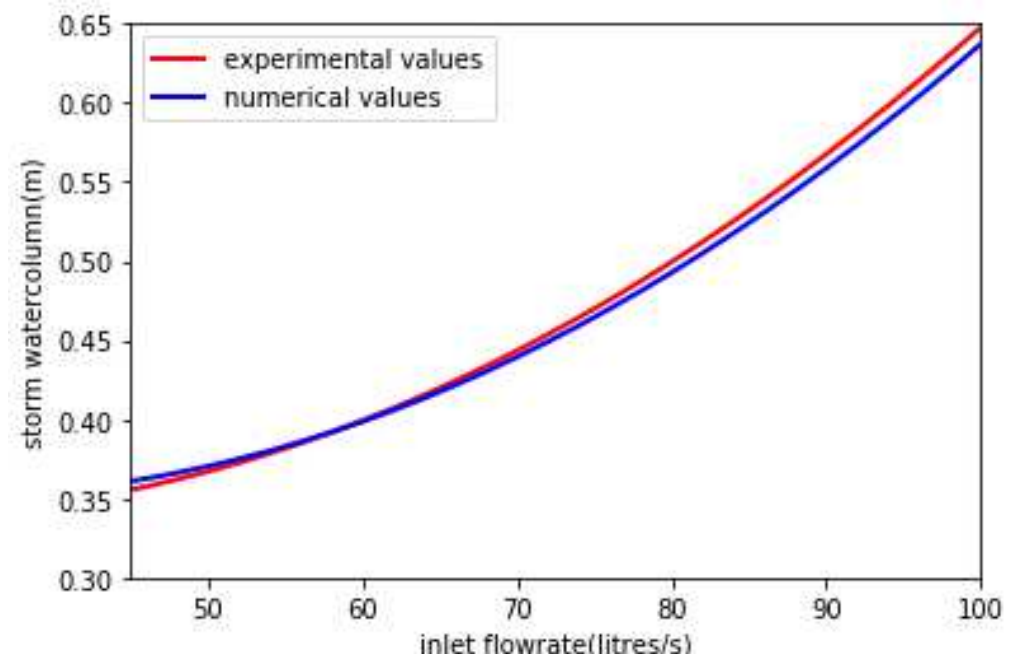

Figure 5: comparison between numerical results of this study and experimental results

\subsection{Velocity field}

The velocity vector of the sewer overflow in the surcharged manhole is shown in figure 6. As can be observed, the velocity vectors are linear at the region of the inlet and outlet pipe, with proper direction from left to right. The linear flow in this region is influenced by the sewer flow that comes directly from the inlet to the outlet pipe. At regions above the inlet and outlet pipes, the velocity vectors take different directions at different places due to the random swirling motion of sewer flow as the storm-water column is developed.

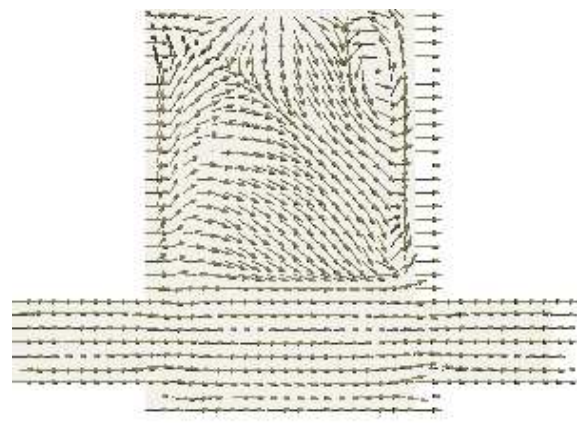

Figure 6: Flow direction

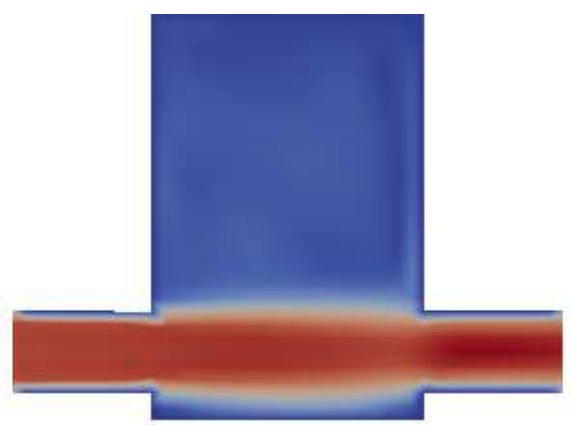

Figure 7: Velocity magnitude

The distribution of velocity magnitude is shown in figure 7. The velocity magnitude is higher at the horizontal region that lies inside the inlet and outlet pipe. The velocity takes its maximum magnitude value in this region due to the direct path of the sewer flow from the inlet to the outlet pipe. At the walls of the pipe, the velocity magnitude decreases compared to the centre of the pipes. This is due to the developed velocity profile of viscous flow on stationary walls. At regions inside the pressure head column, the sewer 
Computational Fluid Dynamics of Manhole Overflow Due to Storm-water Inflow

flow takes different values due to the random motion of the flow as it can be seen by different velocity color distribution.

\subsection{Uplifting forces}

The computation of pressure distribution and the force distribution acting on the manhole cover were carried out using filters from the OpenFOAM visualization tool, paraview 5.4.0. The bottom surface of the manhole cover was extracted independently, for the pressure and force distribution of storm-water, as it was for the surface on the cover that faces the interaction with the hitting storm-water.

The forces acting on the manhole cover are due to the hitting of storm-water on the bottom wall of the cover as its pressure head is rising inside. The rising storm-water column inside the manhole consists of swirling storm currents. Due to the swirling of the storm-water currents, the storm-water hits the cover with different pressure values. Some areas of the cover receive higher values of pressure, causing high-intensity overflow in those directions. As can be inferred from figure 8, the results show those cover areas opposite to the outlet pipe experience the high intense storm overflow.

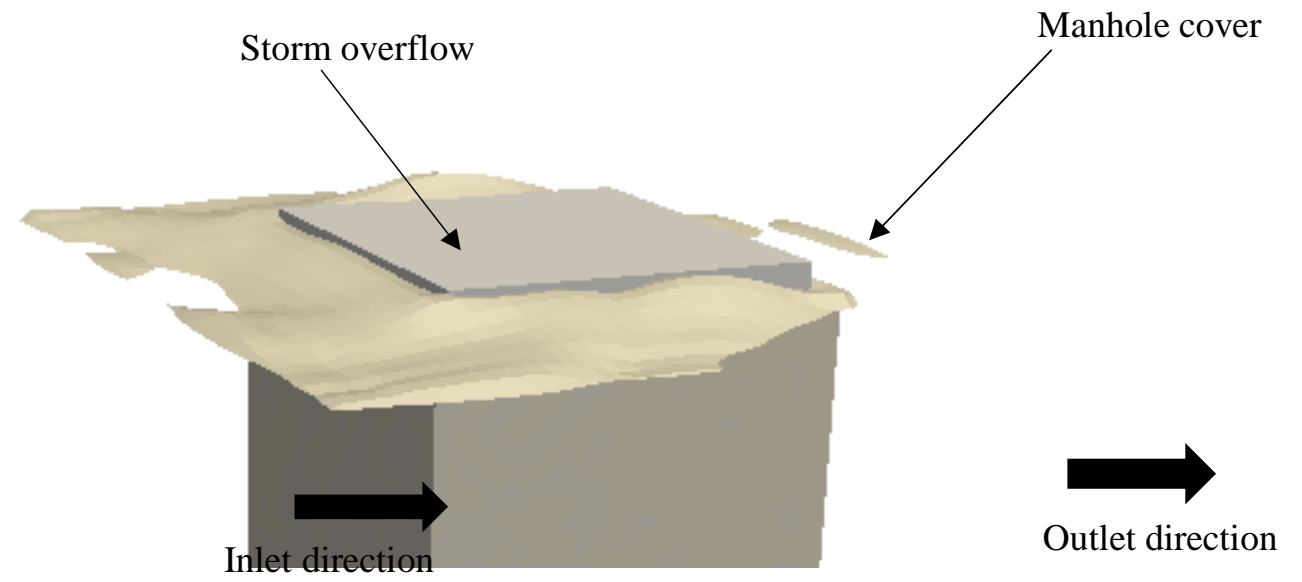

Figure 8: The direction of the intense overflow

This implies that the pressure exerted at the cover is not uniform, and it depends on the velocity direction upon hitting the wall. Figure 9 shows the non-uniform pressure distribution exerted by overflowing storm-water on the manhole cover at the $100^{\text {th }}$ second. The pressure values at this time lie between $78 \mathrm{~N} / \mathrm{m}^{2}$ and $1600 \mathrm{~N} / \mathrm{m}^{2}$. Areas of the cover that face the perpendicular velocity hit with storm-water receive the maximum value of hitting pressure, whereas areas of the cover that storm-water velocity direction is away from it, experience the minimum value of the hitting pressure. 
Sifa Yohana Baseka and Verdiana Grace Masanja

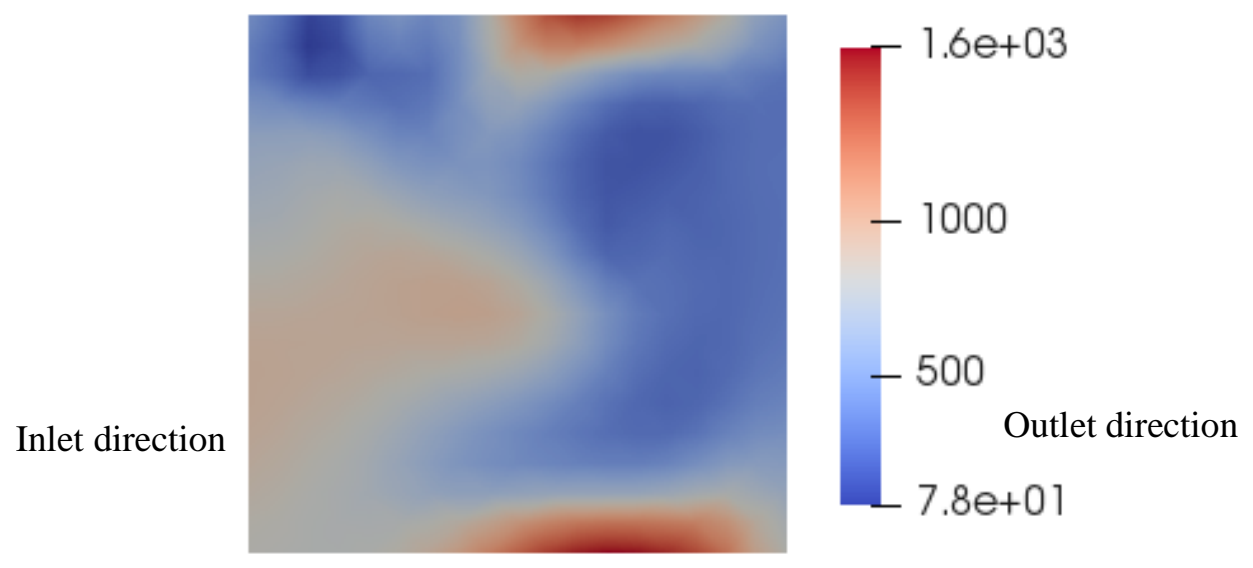

Figure 9:Non-uniform pressure distribution on a manhole cover

Observed in this study is that different pressure distributions acting on the manhole cover result in different force distributions as well. The pressure distribution was then averaged to determine the global pressure (global force) which is, the effective uplifting force that pushes the cover outward. Results of global pressure with time are given in figure 10.

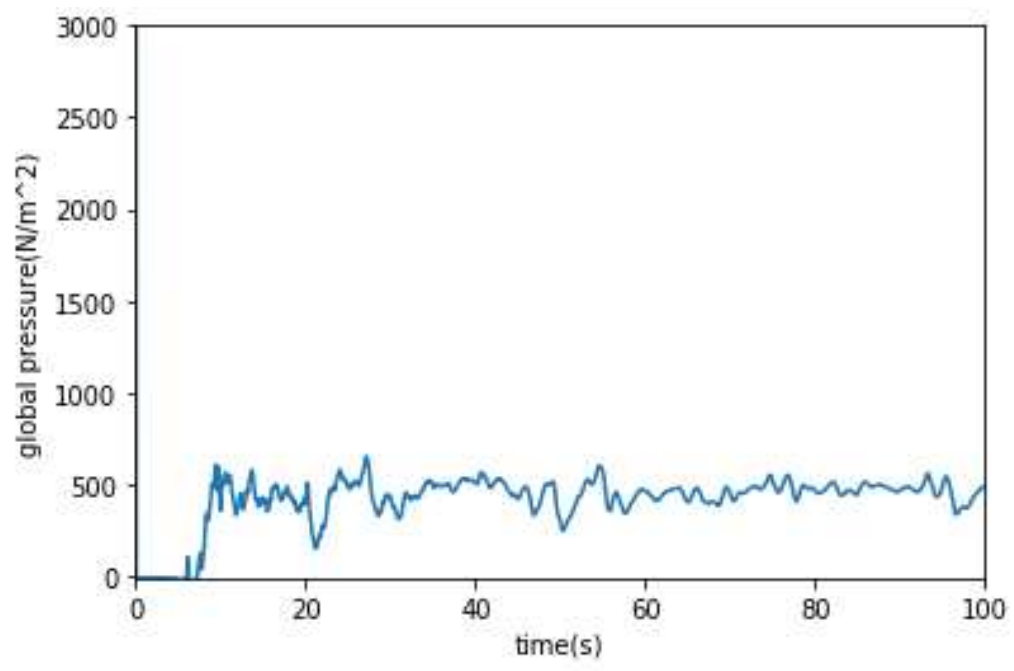

Figure 10: Storm-water global pressure on the manhole cover

It is observed that due to the fluctuation of the pressure field on manhole cover with time, the global pressure also fluctuates with time. Before the overflow occurs, global pressure at the manhole cover increases with time. This can be seen in figure 9 , up to the $10^{\text {th }}$ second. After the storm-water starts overflowing, the global pressure acting on the manhole cover fluctuates about a constant mean. The constant mean global pressure acting on the manhole cover is $415.17 \mathrm{~N} / \mathrm{m}^{2}$. The cross-section area of the manhole 


\section{Computational Fluid Dynamics of Manhole Overflow Due to Storm-water Inflow}

cover is $1 \mathrm{~m}^{2}$. Therefore the global force variation with time is the same as the global pressure variation, as can be inferred from figure 11. At the overflow, the mean constant uplifting force of the storm-water is $415.17 \mathrm{~N}$.

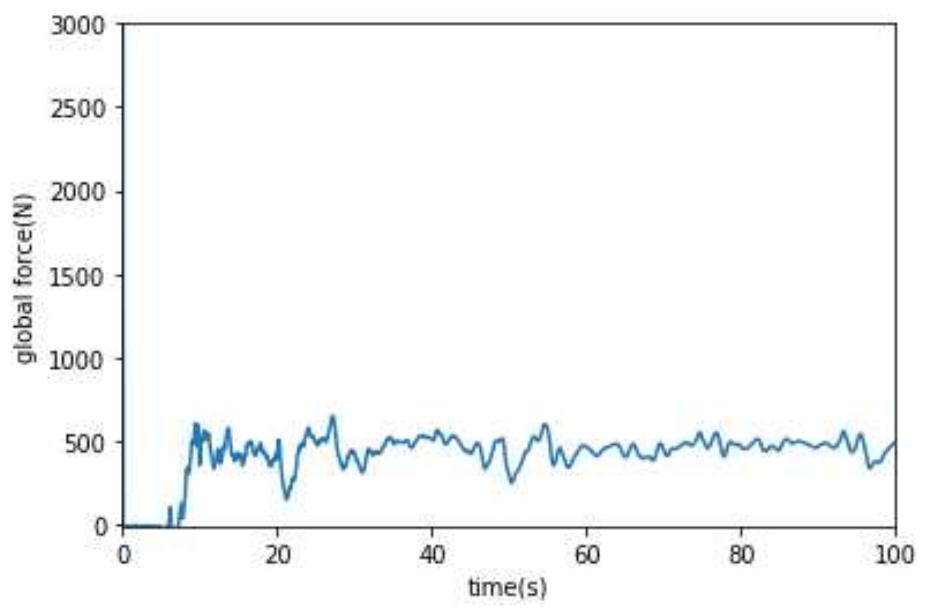

Figure 11: Storm-water uplifting forces

\section{Conclusion}

CFD has produced a realistic situation of manhole overflow as it occurs in a real-life situation. In modelling turbulence, the standard turbulence model, $k-\in$ model, and the volume of the fluid model, a model for phases modelling, together provide a proper prediction of turbulence flow in stormwater flow in sewer systems. The full NavierStokes equations are useful in giving a numerical representation of fluid flow related problems.

The general pressure at the bottom of the manhole increases as the storm-water column inside the manhole, increases. For the overflowed manhole, the highest pressure values are found at the edge which is the intersection between manhole walls and the outlet pipe walls and nearby regions of those edges since in this region, they receive a direct hit of storm-water from the inlet pipe. Velocity magnitude attains its highest values at the horizontal region that lies between the manhole and the inlet and outlet pipes. This is because in this region there is a direct influence of the flow from the inlet pipe to the outlet pipe.

At overflow, there is a different intensity overflow from the manhole. This is because of non-uniform pressure values exerted at manhole cover by the hitting stormwater column inside the manhole. Areas below the manhole cover that are opposite to the outlet pipe give the highest overflow intensity compared to other areas. The global uplifting forces of the rising storm-water column to the manhole cover increase in value until overflow occurs when they remain fluctuating about a constant value. For rectangular manhole with a cross-section area of $1 \mathrm{~m}^{2}$, the uplifting force at the overflow is $415.17 \mathrm{~N}$. 
Sifa Yohana Baseka and Verdiana Grace Masanja

Due to the fact that manhole cover delays the overflow, storm uplifting forces must be determined for each manhole size to establish the required minimum weight of the cover. Manhole cover weights should be greater than the uplifting forces for both rectangular and circular manholes in use.

Acknowledgement. The research was supported by African Development Bank (AfDB). Great tribute to the reviewers of this journal for their appreciable responsibilities on the publication successes.

\section{REFERENCES}

1. J.Chen, S.Wang, Z.Liu and Y. Guo, Network-based optimization modelling of manhole setting for pipeline transportation, Transp. Res. Part E, 113 (2018) 38-55.

2. K.Kaddoura and T.Zayed, Defect- and component-based assessment model for manholes, Tunn. Undergr. Sp. Technol., 82 (2018) 380-393.

3. A.S.Tijsseling, J.G.Vasconcelos, Q.Hou and Z. Bozkus, PVP2019-93086, July 2019.

4. J.Wang, J.Engineering and J.G.Vasconcelos, Investigation of manhole cover displacement during rapid filling of stormwater systems, Journal of Hydraulic Engineering, October 2019.

5. J.B.Jo, J.S.Kim and S.E.Yoon, Experimental estimation of the head loss coefficient at surcharged four-way junction manholes, Urban Water J., 15 (2018) 1-10.

6. M.N.A.Beg, R.F.Carvalho and J. Leandro, Effect of surcharge on gully-manhole flow, Journal of Hydro-environment Research, 19 (2018) 224-236.

7. J.S.Kim, J.B.Jo and S.E.Yoon, Head loss reduction in surcharged four-way junction manholes, Water, 10 (2018) 1741.

8. Z. Zhang and S. C. Chian, Importance of sidewall friction on manhole uplift during soil liquefaction, Soil Dyn. Earthq. Eng., 119 (2019) 51-61.

9. S. C. Xue and G. W. Barton, Incompressible fluid flow simulations with flow rate as the sole information at synthetic inflow and outflow boundaries, Int. J. Numer. Methods Fluids, 78 (2015) 739-760.

10. I.Uddin and M.Karim, Application of volume of fluid (VOF) method for prediction of wave generated by flow around cambered hydrofoil, Procedia Eng., 194 (2017) 82-89.

11. A.Bayon, D.Valero, R.García-bartual and F.Jos, Environmental modelling \& software performance assessment of OpenFOAM and FLOW-3D in the numerical modelling of a low Reynolds number hydraulic jump Vall e, 80 (2016) 322-335.

12. S.Ravelli, G.Barigozzi, F.Pasqua, R.Pieri and R.Ponzini, Numerical and experimental study for the prediction of the steady, three-dimensional flow in a turbine nozzle vane cascade using OpenFOAM, International CAE Conference, (2014) 4-9.

13. F.Moukalled, L.Mangani and M.Darwish, Implementation of boundary conditions in the finite-volume pressure-based method-Part I: Segregated solvers, Numer. Heat Transf. Part B Fundam., 69(6) (2016) 534-562.

14. G.Rocco, G.Coppola and L.De Luca, The VOF method applied to the numerical simulation of a 2D liquid jet under gravity, WIT Trans. Eng. Sci., 69 (2010) 207-217.

15. A.W.Date, Computational Fluid Dynamics, October 2017.

16. M.N.A.Beg, R.Carvalho, P.Lopes, J.Leandro and N. Melo, Numerical investigation of the flow field inside a manhole-pipe drainage system, Hydraul. Struct. Water Syst. Manag. 6th IAHR Int. Symp. Hydraul. Struct., 3706281608(2016) 1-11. 